\title{
RED AND FAR-RED FLUORESCENCE EMISSION RETRIEVAL FROM AIRBORNE HIGH- RESOLUTION SPECTRA COLLECTED BY THE HYPLANT-FLUO SENSOR
}

\author{
S. Cogliati ${ }^{1}$, R. Colombo ${ }^{1}$, M. Celesti ${ }^{1}$, G. Tagliabue ${ }^{1}$, U. Rascher ${ }^{2}$, A. Schickling ${ }^{2}$, P. Rademske ${ }^{2}$, L. \\ Alonso $^{3}$, N. Sabater ${ }^{3}$, D. Schuettemeyer ${ }^{4}$, M. Drusch ${ }^{4}$ \\ ${ }^{1}$ Remote Sensing of Environmental Dynamics Lab., DISAT, University of Milano-Bicocca, Italy; \\ ${ }^{2}$ Institute of Bio- and Geosciences, IBG-2: Plant Sciences, Forschungszentrum Jülich GmbH, Germany; \\ ${ }^{3}$ Department of Earth Physics and Thermodynamics, University of Valencia, Spain; \\ ${ }^{4}$ ESA-ESTEC, The Netherlands;
}

\begin{abstract}
The contribution presents the development and testing of a fluorescence retrieval scheme based on the ESA's FLuorescence EXplorer mission concept. The algorithm employs on a coupled surface-atmosphere forward model at oxygen absorption bands: i) the atmospheric effect is computed by MODTRAN5; ii) the surface reflectance and fluorescence are modeled by means of the Spectral Fitting approach. The algorithm, previously tested on numerical simulations, was further implemented and optimized to process real observations collected by the FLEX airborne demonstrator HyPlant. The retrieval scheme has been tested on a number of flight lines collected in several locations, different ecosystems types, atmospheric conditions and instrument observation conditions. For the first time, this work shows the capability of retrieving canopy fluorescence from real airborne observations by means of a physicallybased algorithm as envisaged for FLEX. The results achieved on the large core data sets of imageries show the consistency of the physical retrieval algorithm for a wide range of scenarios and fluorescence values are in line with ground observations.
\end{abstract}

Index Terms - FLEX, Sun-Induced Chlorophyll Fluorescence, SIF, Spectral-Fitting, HyPlant

\section{INTRODUCTION}

In preparation of the forthcoming ESA's $8^{\text {th }}$ Earth Explorer (EE8) FLuorescence EXplorer mission (FLEX) [1], a series of airborne surveys were carried out since 2012. The campaigns were mainly aimed at developing the FLEX mission concept, including the instrument design and performance analysis, the refinement of processing algorithms and the understanding of the sun-induced fluorescence (SIF) signal in relation with plant photosynthesis for further scientific applications. The highspectral resolution imageries were collected by the airborne imaging spectrometer HyPlant, developed and operated by the Forschungszentrum Jülich (Germany). The comprehensive data set collected is extremely relevant for developing and testing the retrieval algorithm considering different types of ecosystems (e.g., managed crops, natural forests), atmospheric conditions and observational characteristics (i.e. flight altitude etc.). The data sets were complemented with an essential ensemble of ground reference measurements, helpful in evaluating the accuracy of the remote sensing retrievals. The retrieval of fluorescence signal is not trivial due to the fact that this signal represents a very small contribution onto the overall radiance detected by the spectrometer (reflected and emitted). This task is even more difficult in the case of remote sensing observations, from airborne and satellite instruments, because the irradiance spectrum (reference) required in the retrieval must be derived indirectly. Some retrieval approaches make use of non-fluorescence targets within the scene to calibrate the atmospheric effects (scattering and absorption), other approaches cope with this problem employing principal component analysis (PCA) or single vector decomposition (SVD) technique. In this contribution, we present a physically-based retrieval approach based on coupled surface-atmosphere forward model that uses the atmospheric MODTRAN code. The further decoupling of surface radiance and fluorescence from at sensor radiance relies on the Spectral Fitting (SF) approach applied at spectral windows around the main oxygen absorption bands.

\section{MATERIAL AND METHODS}

\subsection{Fluorescence retrieval}

The retrieval algorithm consists of two main components: i) the atmospheric forward model computed by means of MODTRAN5 code; ii) the decoupling of the surface reflected 
radiance and fluorescence achieved based on the Spectral Fitting method. The latter uses general mathematical functions (e.g., polynomials, Voigt etc.) to approximate the surface reflectance and the fluorescence spectral behavior within specific spectral windows around the two telluric oxygen absorption features $\left(\mathrm{O}_{2}-\mathrm{A}\right.$ at $760 \mathrm{~nm} ; \mathrm{O}_{2}-\mathrm{B}$ at 687 $\mathrm{nm})$. The overall forward model used in the fluorescence retrieval derives from the accurate four-stream radiative transfer theory with the addition of the direct and diffuse fluorescence fluxes [2], [3]. It represents an accurate, but at the same time efficient way, for describing the radiative transfer interactions between surface and atmosphere. The retrieval is carried out at-sensor level, comparing the radiance spectra detected by HyPlant with the forward modelled radiance spectrum. In particular, the algorithm computes the at-sensor radiance based on the forward model and optimizes the parameters to obtain the best match with the observed spectra. The atmospheric radiative transfer calculations are based on MODTRAN5 and the atmospheric downward/upward and diffuse/direct transmittance, bidirectional reflectance and the spherical albedo spectra required are derived from an improved version of the MODTRAN Interrogation Technique (MIT). Afterward, the T-14 atmospheric functions are computed according to [2], [3]. The approach developed relies on ancillary measurements to set most of the atmospheric model inputs: the atmospheric status is characterized based on sunphotometer measurements; whereas line-of-sight (LOS) geometry is internally computed from the navigation file. The atmospheric status parameters are considered constant for the entire imagery as commonly used for regular processing of airborne imageries. The atmospheric measurements were normally collected by using the Microtops II sun-photometer or whenever available the Cimel instruments belonging the Aeronet network.

The flow-chart of the computer code implemented to retrieve fluorescence from HyPlant imagery is reported in Figure 1. Following the regular input/output data handling operations, the core of the processing function consists of few fundamental steps: (i) calculation of the $\mathrm{T}-14$ atmospheric functions; (ii) in-flight instrument spectral characterization (SPECCAL) based on a modified version of [4]; finally, (iii) retrieval of fluorescence/reflectance. The left-side entries show the input files requested by the processing code: HyPlant imagery, navigation file and atmospheric input parameter file. The right-side exits indicate the output files generated by the processing code that include the atmospheric function spectra, the imaging spectrometer characterization in the across-track direction and, finally the fluorescence and reflectance maps.

Finally, the image processing was facilitated by the implementation of techniques such as vectorization and parallel computing on shared memory in the retrieval software code. In fact, the computer code can be executed on High-Performance Computing (HPC) infrastructure allowing to process large airborne data sets efficiently.

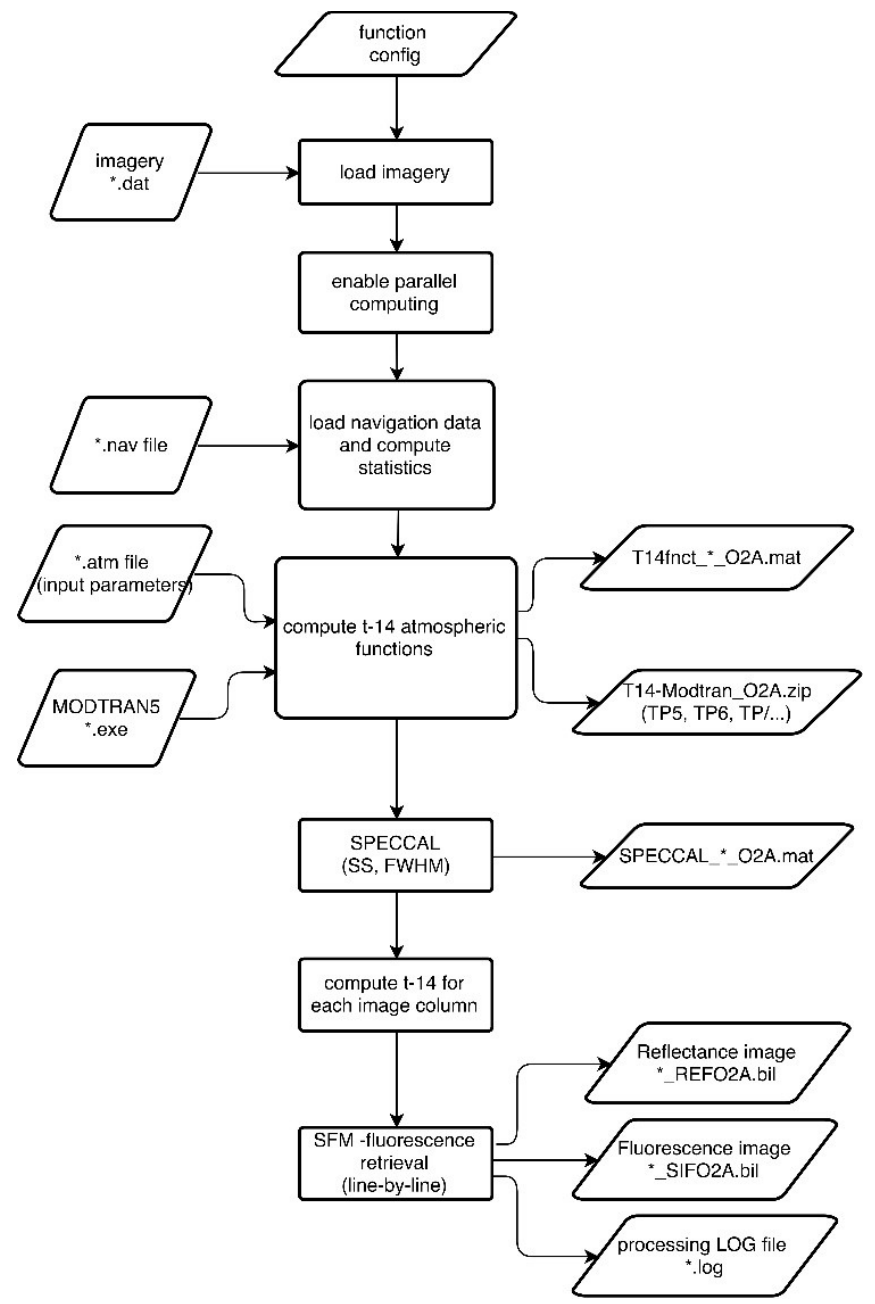

Fig. 1: Flow-chart of the fluorescence retrieval algorithm implemented for HyPlant imaging spectrometer.

\subsection{Airborne imagery - HyPlant}

The HyPlant sensor is a high-performance imaging airborne spectrometer. It consists of two sensor heads [5]. The DUAL module is a line-imaging push-broom hyperspectral sensor, which provides contiguous spectral information from 370 to $2500 \mathrm{~nm}$ in one device utilizing a common fore objective lens with $3 \mathrm{~nm}$ spectral resolution in the VIS/NIR spectral range and $10 \mathrm{~nm}$ spectral resolution in the SWIR spectral range. The vegetation fluorescence signal is measured with a separate push-broom sensor, the FLUO module, which produces data at high spectral resolution (FWHM about 0.25 $\mathrm{nm}$ ) in the spectral region of the two oxygen absorption bands. The pre-processing of airborne imageries includes a number of standard processing steps typical of image spectroscopy instruments such as the dark-current correction, the spectral and radiometric calibration, the non-linearity correction and the deconvolution of the instrument point spread function (PSF). A core dataset of flight-lines was selected from the total ensemble of observations recorded during summer 2015 and 2016 in Germany, Italy and Poland. 
It represents an important testing-bench to assess the overall retrieval algorithm performance because selected imageries were collected in various locations characterized by different types of canopy (managed crops and natural vegetation), during different measurement days and different atmospheric conditions. The imageries were typically collected at three different flight-altitude above the surface 350-600-1800 meters, that correspond to pixel size of $0.5-1.0-3.0$ meters respectively.

\section{EXPERIMENTAL RESULTS}

The fluorescence maps at $\mathrm{O}_{2}-\mathrm{A}$ and $\mathrm{O}_{2}-\mathrm{B}$ bands are reported and discussed in this section. A first evaluation has been carried on by using radiometrically and spectrally calibrated imagery, according to the standard instrument processing chain. The direct comparison of ground-truth values and airborne fluorescence map is the preferred way to have an overall assessment of the entire airborne processing chain. However, intermediate processing products can be analyzed to gain information on specific processing routines (modules). One way involves the evaluation of the atmospheric model input parameters used in processing the HyPlant imagery trough the comparison of modelled irradiance at ground level against the field spectroscopy measurements. In fact, the T-14 atmospheric functions can be combined not only to simulate the at-sensor the radiance spectrum, but it can be modified to compute the irradiance spectrum at ground from Equations (1) and (2).

$$
\begin{aligned}
L_{S U N} & =\frac{E_{s}^{0} \cos \theta_{s}}{\pi} \tau_{s s} \\
L_{S K Y} & =\frac{E_{S}^{0} \cos \theta_{s}\left(\tau_{s d}+\tau_{s s} \rho_{d d} \overline{r_{s d}}\right)+\rho_{d d}}{1-\overline{r_{d d}} \rho_{d d}}
\end{aligned}
$$

The $E_{S}^{0}$ is the extra-terrestrial solar irradiance, $\theta_{S}$ the local solar zenith angle, $\tau_{s s}$ the direct atmospheric transmittance in the direction of the sun, $\tau_{s d}$ the diffuse atmospheric transmittance for solar incidence and $\rho_{d d}$ the spherical albedo. The $\overline{r_{s d}}$ and $\overline{r_{d d}}$ are the directional-hemispherical reflectance and the bi-hemispherical reflectance respectively. As an example, Figure 2 shows a comparison between the theoretical spectrum simulated by the atmospheric model and the field spectroscopy observations. Currently, the analysis was performed on the radiance spectra with the lower spectral resolution because they cover the entire VNIR spectral range (350-1000 nm). It allows to better analyze the atmospheric modeling on a broader spectral range, including the blue region which is more informative about the atmospheric scattering. However, future analysis will involve also the high-spectral resolution measurements at the oxygen absorption bands, but it will require a proper knowledge of the field spectrometer instrument spectral response function.
A similar evaluation of intermediate-products involved the characterization of the instrument FWHM and spectral shift in the imagery spatial domain (across-track). In fact, not accurate characterization of the atmospheric parameters can severely affect the $\mathrm{O}_{2}$ bands depth, introducing errors in the vicarious characterization of the instrument spectral performance. The results obtained for the data sets analyzed indicate a general robustness for the different scenarios. The further evaluation of the retrieval algorithm performance involves the analysis of the final fluorescence maps at the $\mathrm{O}_{2}$ A and $\mathrm{O}_{2}-\mathrm{B}$ bands. A typical example of the results currently achieved on the HyPlant imagery are reported in Figure 3.

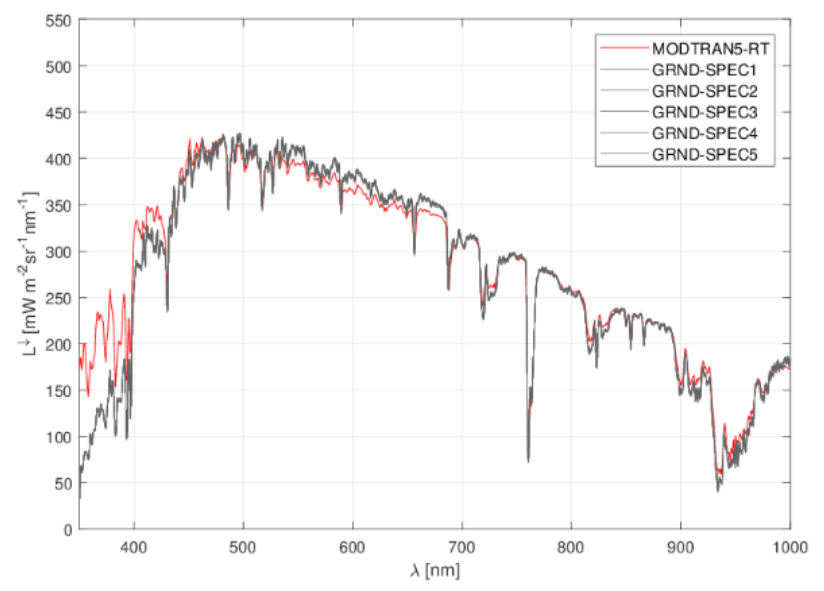

Fig. 2: Comparison of irradiance spectrum computed by MODTRAN5 (red) vs. simultaneous field spectroscopy measurements (gray) collected on a calibrated white reference panel by VNIR spectrometer (5 samples simultaneous to HyPlant).

The overall magnitude of SIF values is close to the expected values for both the oxygen absorption bands $\left(0-2.5 \mathrm{mWm}^{-2} \mathrm{sr}^{-}\right.$ $\left.{ }^{1} \mathrm{~nm}^{-1}\right)$, but probably fluorescence values are slightly overestimated considering that imagery was collected in the late afternoon. The spatial patterns within the image are meaningful: vegetation shows larger fluorescence values, while roads and bare soils have lower values, close to zero. On average, healthy and dense agricultural fields have maximum fluorescence values in the range between 1.8-2.5 $\mathrm{mWm}^{-2} \mathrm{sr}^{-1} \mathrm{~nm}^{-1}$ at $\mathrm{O}_{2}-\mathrm{A}$ band and 1.4-2.0 $\mathrm{mWm}^{-2} \mathrm{sr}^{-1} \mathrm{~nm}^{-1}$ at $\mathrm{O}_{2}$-B band. Other fields with lower amount of biomass amount have slightly lower fluorescence values that range 1.0-1.7 and $0.9-1.5 \mathrm{mWm}^{-2} \mathrm{sr}^{-1} \mathrm{~nm}^{-1}$ for far-red and red fluorescence respectively. The spatial behavior of the far-red fluorescence map looks sharp (low noise) and even small geometric features can be clearly observed, for example roads are well defined as well as small paths inside agricultural crops fields. The red fluorescence retrieved at the $\mathrm{O}_{2}-\mathrm{B}$ band shows a higher level of noise, in fact values of homogeneous fields appear more scattered around the average value than far-red fluorescence. Similar and consistent results were 
achieved for all the core dataset flight lines covering similar crops areas and natural forest ecosystems.

However, one of the issues in processing high-resolution spectroscopy imageries collected by HyPlant, concerns the instrument PSF. Currently, a deconvolution algorithm has been included in the HyPlant pre-processing chain, but the performances of the module are still under evaluation. Further investigations are needed to better understand the PSF deconvolution algorithm performance implemented in HyPlant pre-processing chain, in particular in relation with the Spectral Fitting retrieval.

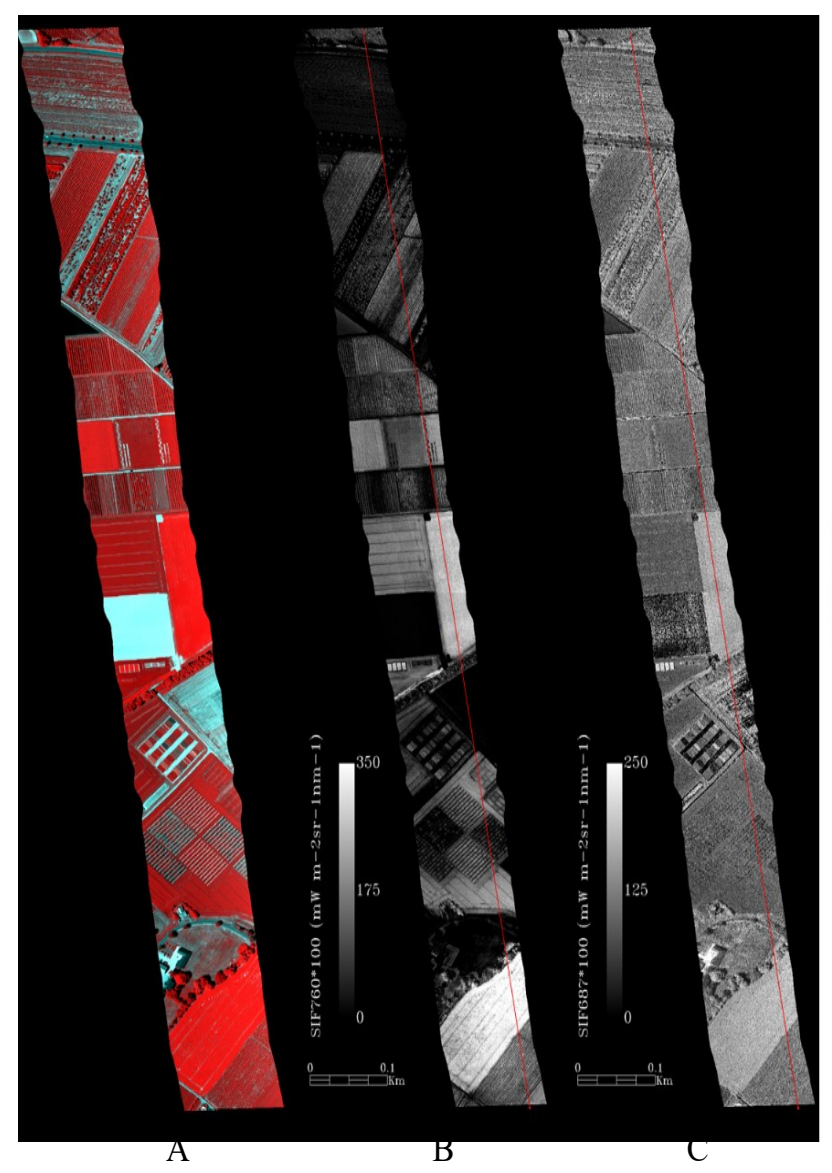

Fig. 3: Imagery 20150630-SOY-1536-0350-L6-N collected on June 30th at 15:36 local time. (A) false color composite (R: $787 \mathrm{G}: 125 \mathrm{~B}:$ 58); (B) far-red fluorescence retrieved at $\mathrm{O}_{2}-\mathrm{A}(760 \mathrm{~nm})$; (C) red fluorescence retrieved at $\mathrm{O}_{2}-\mathrm{B}(687$ $n m)$.

\section{CONCLUSIONS}

A physically-based fluorescence retrieval algorithm based on the retrieval algorithm concept of the FLEX mission has been developed and optimized for processing real airborne observations. An extensive testing was conducted on a coredata set which consists of several imageries collected in several locations, different ecosystems and atmospheric conditions. Intermediate products such as surface irradiance and in-flight instrument characterization were made available permitting to evaluate wherever possible the performance of single processing modules (or even smaller parts of the processing chain). This part is essential to understand the contribution of specific routines to the total retrieval error budget. The fluorescence maps obtained in different scenarios (i.e., locations, canopies, atmospheres etc.) show consistent behavior in terms of spatial patterns and absolute red and far-red fluorescence values. The PSF deconvolution algorithm was essential to remove the filling-in effect caused by the instrument to retrieve consistent values either on vegetation and non-fluorescence targets. The deconvolution algorithm currently available greatly supported the understanding of PSF effect on fluorescence maps, however it introduces a significant amount of noise that severely affects final red fluorescence map at $\mathrm{O}_{2}-\mathrm{B}$ band.

\section{ACKNOLOWDGMENT}

This work has been developed in the framework of several airborne campaigns founded by the European Space Agency (ESA) under the Contract No. 4000107143/12/NL/FF/If CCN3 and Contract No. 4000107143/12/NL/FF/If CCN4.

\section{REFERENCES}

[1] M. Drusch et al., "The FLuorescence EXplorer Mission Concept-ESA's Earth Explorer 8," IEEE Trans. Geosci. Remote Sens., vol. PP, no. 99, pp. 1-12, 2016.

[2] W. Verhoef, C. van der Tol, and E. M. Middleton, "Hyperspectral radiative transfer modeling to explore the combined retrieval of biophysical parameters and canopy fluorescence from FLEX - Sentinel-3 tandem mission multi-sensor data," Remote Sens. Environ., Oct. 2017.

[3] S. Cogliati et al., "Retrieval of sun-induced fluorescence using advanced spectral fitting methods," Remote Sens. Environ., vol. 169, pp. 344-357, Nov. 2015.

[4] M. Meroni et al., "Characterization of fine resolution field spectrometers using solar Fraunhofer lines and atmospheric absorption features.," Appl. Opt., vol. 49, no. 15, pp. 2858-2871, May 2010.

[5] U. Rascher et al., "Sun-induced fluorescence - a new probe of photosynthesis: First maps from the imaging spectrometer HyPlant," Glob. Chang. Biol., vol. 21, no. 12, pp. 4673-4684, Jul. 2015. 